\title{
Deceptive jamming method for countering ISAR based on electromagnetic scattering property
}

\author{
Ning Tai ${ }^{\dagger}$, Chao Wang and Nai-Chang Yuan, \\ College of Electronic Science and Engineering, \\ National University of Defense Technology, Changsha, 410073, China \\ E-mail: 358041578@qq.com \\ Li-Guo Liu, \\ College of Electronic Engineering, Naval University of Engineering, \\ Wuhan, 410073, China \\ E-mail:poorthinker@sina.com \\ Long Xiong \\ Unit 61768 of PLA, Sanya, 572000, China \\ E-mail:xljohhn1990@126.com
}

\begin{abstract}
A frequency domain deceptive jamming method is proposed against ISAR based on the electromagnetic property. The RCS of a 3-D vessel model is computed to build up the target template. The template is used to modulate the intercepted radar signal in frequency domain. Containing the electromagnetic scattering property of the target, the ISAR image induced by the jamming signal is similar to a real one. The proposed method reduces the computation load and successively reflects the target information. The correctness and effectiveness of the proposed method is validated by simulation results. The method shows superiority in real-time property and convenience for engineering implementation.
\end{abstract}

Keywords: Electromagnetic model; RCS; ISAR; Deceptive jamming; LFM.

\section{Introduction}

Radar jamming and anti-jamming technology have been the hot topics in the field of electronic countermeasure for several decades. The development in jamming technology improves the method of anti-jamming and vice versa [1,2]. Inverse synthetic aperture radar, a day-and-night, all-weather, powerful coherent radar, is widely applied in many fields. Widely speaking, the jamming method for countering ISAR can be classified into active jamming and passive jamming. According to the jamming effects, active jamming consists of depressing jamming (noise jamming) and deceptive jamming (false target jamming) $[3,4]$. 
Being able to achieve partial processing gain, the coherent noise jamming method can effectively jam the radar with a lower power [5]. The deceptive jamming aims at creating several false targets to confuse the radar operator. The classical algorithms include the convolution modulation method [6], digital image synthesizer [7], etc.

In this paper, we compute the RCS of the 3-D vessel model to build up the template. The RCS template can reflect the variation of the structure, the amplitude of the scattering parts and the shading effect caused by target's movement. The jamming method can induce a false ISAR image .

\section{Theory of Deceptive Jamming Method}

\subsection{Computation of the target's attitude angle}

At any time, the target's attitude angle with respect to the light of sight (LOS) of radar signal shall be obtained. As shown in Fig. 1, the Cartesian coordinate system $o x y z$, with its origin locating at the location of ISAR, is set up. At time 0 , the jammer is located $R_{0}$ away from ISAR and moves along the y-axis with a velocity of $V_{J}$. Suppose that the coordinate of the jammer is $\left(x_{J 0}, y_{J 0}, z_{J 0}\right)$, the distance $R_{0}$ satisfies

$$
R_{0}=\sqrt{x_{J 0}^{2}+y_{J 0}^{2}+z_{J 0}^{2}}
$$

The included angle between LOS and the negative position of $\mathrm{z}$-axis is defined as $\theta$. The angle between the projection of LOS onto the xoy plane and the positive position of $\mathrm{x}$-axis is defined as $\varphi$.

$$
\theta=\cos ^{-1}\left(-\frac{z_{J 0}}{\sqrt{x_{J 0}^{2}+y_{J 0}^{2}+z_{J 0}^{2}}}\right), \varphi=\cos ^{-1}\left(\frac{x_{J 0}}{\sqrt{x_{J 0}^{2}+y_{J 0}^{2}}}\right)
$$

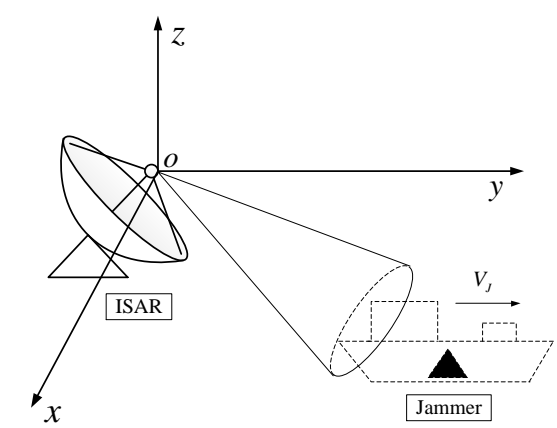

Fig. 1. The scene of the proposed jamming method. 
According to (1) and (2), the azimuth angle $\varphi$ and pitching angle $\theta$, which describe the attitude of the jammer, can be computed. The attitude angle is used to select the corresponding RCS template.

\subsection{Template of the false target}

When computing the RCS, we should consider both the frequency of the radar signal and the attitudes. Normally, the bandwidth of the radar signal is not very large in case that the target will occupy too many range cells. So, during the computation of RCS, the center frequency is $10 \mathrm{GHz}$ and the bandwidth is 300 $\mathrm{MHz}$, which is suitable for creating a false vessel target.

\subsection{Modulation of electromagnetic scattering property}

Suppose that the ISAR transmit an LFM signal with the bandwidth, the pulse width defined as $B$ and $T$, respectively. The frequency of the carrier wave is $f_{c}$. The expression of the LFM signal is

$$
s(t)=\operatorname{rect}\left(\frac{t}{T}\right) \cdot \exp \left(j 2 \pi\left(f_{c} t+\frac{1}{2} k t^{2}\right)\right.
$$

where $t$ is time variable and $k$ is chirp rate. When $|t| \leq T / 2, \operatorname{rect}(t / T)$ is 1 and otherwise yields 0 . If the jammer directly transmits the intercepted radar signal, the jamming signal can be

$$
s_{r}(t)=\operatorname{rect}\left(\frac{t-2 R_{J}(t) / c}{T}\right) \cdot \exp \left(j 2 \pi\left(f_{c}\left(t-\frac{2 R_{J}(t)}{c}\right)+\frac{1}{2} k\left(t-\frac{2 R_{J}(t)}{c}\right)^{2}\right)\right.
$$

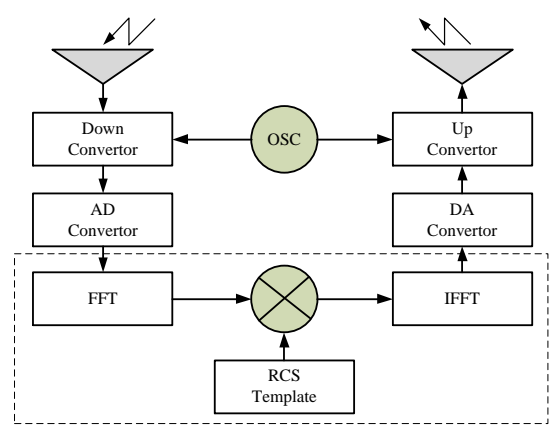

Fig. 2. Workflow of the proposed method.

In the detection stage, the jammer analyzes the frequency, pulse width, bandwidth of the radar signal. After obtaining the radar signal, the jammer turns into the jamming stage. The jamming signal is achieved through the multiplication of the RCS template and the radar signal (in frequency domain), as shown in Fig.2. Then the jamming signal is transformed into time domain. 


$$
s_{j}\left(t, t_{m}\right)=F F T^{-1}\left[F F T\left(s_{r}(t)\right) \cdot R C S(f, \theta, \phi)\right]
$$

where $\operatorname{RCS}(f, \theta, \phi)$ is the extraction result of the whole template.

\subsection{Characteristic of the jamming signal}

The radar echo of the target can be described through a linear system where the input, output signal are the radar signal and the target echo, respectively. Then the target echo can be

$$
s_{e}(t)=\int_{y_{\min }}^{y_{\max }} \int_{\Omega} s(t-2 y / c) \cdot \sigma(x, y) d x d y
$$

where $y$ stands for the distance from certain scatterer to the radar. $\sigma(x, y)$ is the back scattering coefficient. $\Omega$ denotes the projection of the target along the azimuth direction. We can rewrite the target echo after several deviations.

$$
\begin{aligned}
s_{e}(t) & =\int_{y_{\min }}^{y_{\max }}\left(s(t) \otimes \int_{\Omega} \delta(t-2 y / c) \cdot \sigma(x, y) d x\right) d y \\
& \left.=\int_{y_{\min }}^{y_{\max }}(s(t) \otimes(\delta(t-2 y / c)) \cdot \sigma(y))\right) d y \\
& =s(t) \otimes \int_{y_{\min }}^{y_{\max }} \delta(t-2 y / c) \cdot \sigma(y) d y=s(t) \otimes h(t)
\end{aligned}
$$

where $h(t)$ is the response function of the system, $\otimes$ is the process of convolution. So (5) can be rewritten into (8), where HRRP means the 1-D high resolution range profile.

$$
s_{j}\left(t, t_{m}\right)=s_{r}(t) \otimes F F T^{-1}(R C S(f, \theta, \phi))=s_{r}(t) \otimes H R R P
$$

\section{Simulations}

This section presents the jamming effect of the method. The ISAR operates at X band and the carrier frequency is $10 \mathrm{GHz}$. The transmitted signal is the LFM signal with pulse width of 1 us and $20 \mathrm{us,} \mathrm{bandwidth} \mathrm{of} 100 \mathrm{MHz}$ and $300 \mathrm{MHz}$. The remaining parameters are listed in Tab.1.

Table 1. Simulation parameters.

\begin{tabular}{lll}
\hline Name & Value & Unit \\
\hline Jammer Velocity & 100 & $\mathrm{~m} / \mathrm{s}$ \\
Observation Time & 0.256 & $\mathrm{~s}$ \\
Jammer Location & $(750,433,-500)$ & $\mathrm{m}$ \\
ISAR Location & $(0,0,0)$ & $\mathrm{m}$ \\
PRF & 1000 & $\mathrm{~Hz}$ \\
\hline
\end{tabular}


The radar signal and the RCS template are shown in Fig. 3, where we see that the frequency span of the template is $500 \mathrm{MHz}$. We simulate the jamming signal and use Range-Doppler algorithm to process the imaging procedure.

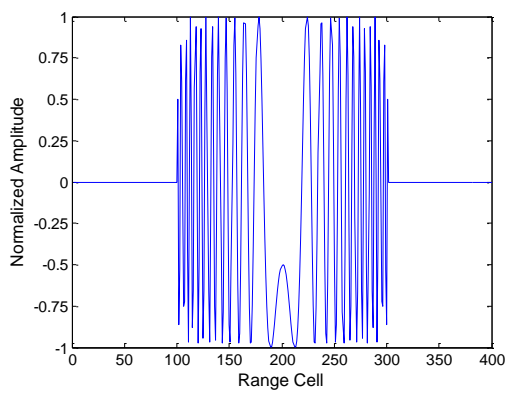

(a) The transmitted LFM signal in time domain

Fig. 3. The radar signal and the template of false target.

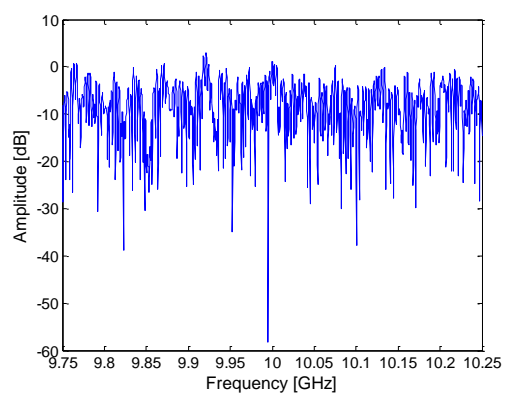

(b) The RCS template of the false target.

Firstly we analyze the situation of $100 \mathrm{MHz}$ bandwidth and 1 us pulse width The ISAR image is shown in Fig.4, where Fig.4(a) presents the 1-D range profile result. The 2-D ISAR image is shown in Fig.4(b). From the figure we see that the main scatterers locate at the body and the top of the vessel. The scatterers with lower amplitude surround the outline of the vessel.

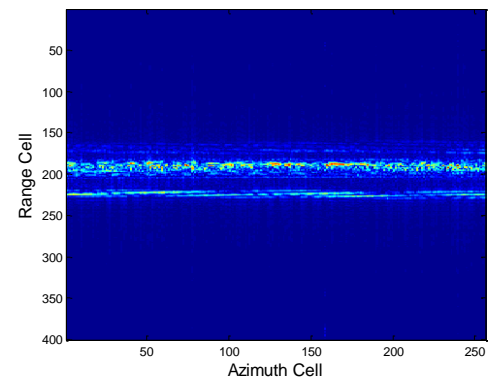

(a) 1-D range profile of the false target Fig. 4. Range-Doppler ISAR image of the target when bandwidth $=100 \mathrm{MHz}$, pulse width $=1$ us.

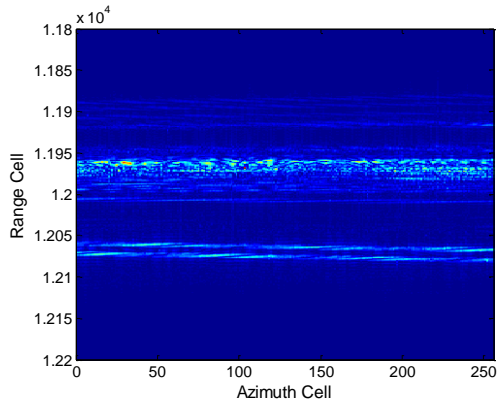

(a) 1-D range profile of the false target.

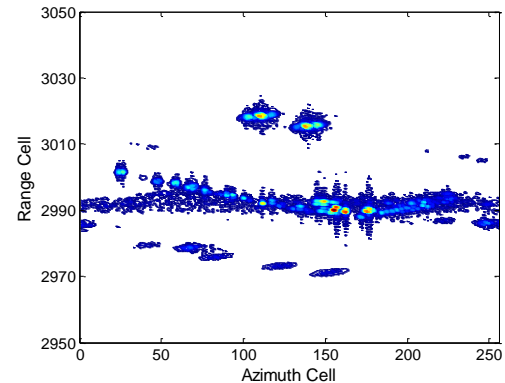

(b) ISAR image of the false target.

Fig. 5. Range-Doppler ISAR image of the target when bandwidth $=300 \mathrm{MHz}$, pulse width $=20 \mathrm{us}$ 
Next we increase the bandwidth and the pulse width of the LFM signal to test the adaptability of the jamming method. An LFM signal with larger bandtime product provides more processing gain and higher resolution. From Fig.5 we see that more details of the vessel are shown and the SNR of the image improves. The jamming method is able to create the jamming signal against different radar signals. When ISAR tracks the false target, the threat to our real target is descended.

\section{Conclusions}

This paper proposes a method of creating a 2-D false target in frequency domain. The jamming signal reflects the scattering property of the target. The main procedure of the method contains FFT, multiplication and IFFT. The proposed method is of good adaptability and real time performance, which is suitable for engineering implementation.

\section{References}

1. Y.P. Zhu, Y.H. Zhang, H.Q. Wang and X. Li, Research on anti-jamming of inverse synthetic aperture radar based on slope-varying LFM signal, Signal Processing 26, 417 (2010).

2. J.K. Zhang, S.Q. Xing, D.H. Dai, Y.Z. Li and S.P. Xiao, Three-dimensional deceptive scene generation against single-pass InSAR based on coherent transponders, IET Radar Sonar Navig.10, 477 (2016).

3. Y. Zhang, S.Q. Yang and W.X. Zheng, Pulse weighted frequency modulation jamming technique countering ISAR, Systems Engineering and Electronics30, 249 (2008).

4. X.D. He, J. Zhu, J. Wang, D.P. Du and B. Tang, False Target Deceptive Jamming for Countering Missile-Borne SAR, in Proc. IEEE Int. Conf. on Computational Science and Engineering (CSE), (Chengdu, China, 2014).

5. N. Tai, Y.J. Pan and N.C. Yuan, Quasi-coherent noise jamming to LFM radar based on pseudo-random sequence phase-modulation, Radioengineering24, 1013 (2015).

6. X.F. Zhang, H.B. Tang and S.K. Xu, A novel algorithm of deception jamming signal with ISAR radar, Journal of National University of Defense Technology36, 49 (2014).

7. D. J. Fouts, P. E. Pace, C. Karow and S.R.T. Ekestorm, A single-chip false target radar image generator for countering wideband imaging radars, IEEE J. Solid-State Circuits37, 751 (2002). 